Check for updates

Cite this: RSC Adv., 2017, 7, 36728

Received 29th March 2017

Accepted 20th July 2017

DOI: $10.1039 / \mathrm{c} 7 \mathrm{ra03632a}$

rsc.li/rsc-advances

\section{Pharmacokinetics study of isorhamnetin in rat plasma by a sensitive electrochemical sensor based on reduced graphene oxide $\uparrow$}

\author{
Huaping Peng, $t^{\text {ac }}$ Lingling Zhang,,$^{\text {ac }}$ Zhili Cai, ${ }^{\text {ace }}$ Yanhong Wu, ${ }^{\text {ac }}$ Namin Chen, ${ }^{\text {ac }}$ \\ Chao Gu, ${ }^{a c}$ Yuanzhong Chen, ${ }^{\star a b}$ Xinhua Lin, ${ }^{\star a c}$ Xinghua Xia (D) ${ }^{d}$ and Ailin Liu (D) *ac
}

Isorhamnetin (ISO), a flavonol aglycone, plays an important role in human health, such as cardiovascular protection, anti-allergy activity, anti-inflammatory effects, and anticancer activity. Thus, the highly sensitive and rapid detection of ISO, and its pharmacokinetic study, has important clinical significance in the field of medicine. Herein, a simple, rapid, sensitive, and accurate method for the electrochemical determination of isorhamnetin (ISO) for pharmaceutical quality control and pharmacokinetic research was developed using an electrochemically reduced graphene oxide modified glassy carbon electrode (ERGO/GCE). The results indicated that the ERGO/GCE remarkably increased the peak currents of ISO oxidation. Under optimized conditions, using differential pulse voltammetry (DPV), the peak current had a linear dependence on the concentration of ISO in the range of $1.0 \times 10^{-8}$ to $1.2 \times 10^{-5} \mathrm{M}$ with a low detection limit of $3.2 \times 10^{-9} \mathrm{M}$. The present voltammetric method has been successfully applied to the detection of ISO in tablets and in plasma. Moreover, this assay has also been successfully applied in the pharmacokinetic study of ISO in vivo through intragastric administration of tablets to SD-rats. Therefore, this strategy opens up a new avenue for the application of an electrochemical method in the field of ISO pharmacokinetics.

\section{Introduction}

Isorhamnetin (ISO) is an important member of the flavonoid family from Hippophae rhamnoides L. and the Ginkgo biloba L. Studies have shown that ISO acts as a powerful antioxidant and is capable of mopping up free radicals, the harmful molecules linked to cancer, hence ISO has good anti-cancer activity. ${ }^{1}$ Furthermore, it also has a significant effect on relieving angina and decreasing the content of cholesterol in serum. In clinical practice, ISO plays an important role in suppressing the aggregation of platelets, and it appears to cause many biochemical and pharmacological effects on human health. ${ }^{2}$ Thus, it is imperative to

${ }^{a}$ Department of Pharmaceutical Analysis, Faculty of Pharmacy, Fujian Medical University, Fuzhou 350108, China. E-mail: xhl1963@sina.com; Chenyz@mail.jmu. edu.cn; ailinliu@mail.fjmu.edu.cn; Fax: +86 591 22862016; Tel: +8659122862016

${ }^{b}$ Fujian Institute of Hematology, The Affiliated Union Hospital of Fujian Medical University, Fuzhou 350000, China

'The Higher Educational Key Laboratory for Nano Biomedical Technology of Fujian Province, Fujian Medical University, Fuzhou 350108, China

${ }^{d}$ State Key Laboratory of Analytical Chemistry for Life Science, School of Chemistry and Chemical Engineering, Nanjing University, Nanjing 210023, China

${ }^{e}$ Department of Pharmacy, Jinjiang Hospital, Quanzhou, 362200, China

$\dagger$ Electronic supplementary information (ESI) available. See DOI: $10.1039 / \mathrm{c} 7 \mathrm{ra03632a}$

$\ddagger$ Huaping Peng and Lingling Zhang contributed equally to the present study. establish a rapid, simple and convenient method for the determination of ISO in tablets or in serum samples.

Up to now, many methods have been developed for the determination of ISO, including high performance liquid chromatography (HPLC), ${ }^{3}$ gas chromatography (GC), ${ }^{4}$ capillary electrophoresis $(\mathrm{CE})^{5}$ and a quartz microchip ${ }^{6}$ coupled with various detection techniques, such as UV spectrophotometry. ${ }^{7}$ The coupling of these techniques may provide high selectivity of the assay, but brings also some disadvantages of operating complexity, time and reagent consumption, and high cost, which hamper their further application. In contrast to these methods, electrochemical techniques have been extensively used as nontedious procedures, accurate, with inexpensive instruments, less time consumption, highly selective and sensitive method of analysis. ${ }^{8-10}$ As we know, flavonoids and related polyphenols, including ISO, generally consist of two benzene rings linked by an oxygen containing heterocycle. Meanwhile, the oxidation reaction of flavonoids is strongly related to the structure of the flavonoids, which contains several free phenolic hydroxyl groups. Therefore, electrochemical methods had been widely used to determine flavonoids. ${ }^{11-14}$ Furthermore, it is worth mentioning that the electrochemical behavior of ISO at bare glassy carbon electrode (GCE) has been studied by our group. ${ }^{15}$ However, the sensitivity and detection range of the analytical methods should be improved, and it is of great importance to study the further application of the presented approach. 


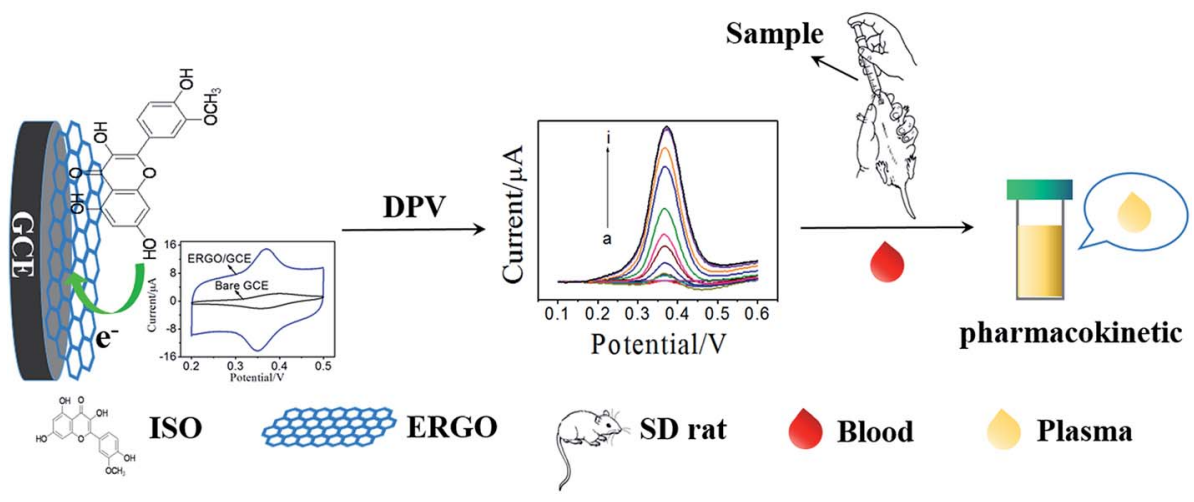

Scheme 1 Scheme of ISO detection and its pharmacokinetics investigation.

Graphene, a two dimensional carbon-based nanomaterials, has recently received extensive interest in the fields of electrochemical sensors and biosensors due to their intrinsic properties, including high surface-to-volume ratio, ease of functionalizing its surface, excellent conductivity, and biocompatibility. ${ }^{16-18}$ Additionally, graphene-based materials modified films can significantly improve the electrocatalytic properties of substrates, decrease the overpotential, increase the reaction rate and improve the stability and reproducibility of the electrode response. Thus, graphene-based materials modified electrodes have been widely used in the determination of biomolecules, drugs, smallmolecules, and other organic species. ${ }^{16,19-22}$

Pharmacokinetic studies on active ingredients in natural products and traditional Chinese medicines are important to illustrate their action mechanism..$^{23,24}$ Thus, developing a facile and reliable method to investigate the pharmacokinetic of ISO is imperative. In the present work, a sensitive electrochemical sensor for ISO detection was fabricated using graphene modified GCE, which was prepared by electrochemical reduction of graphene oxide (ERGO). Subsequently, the electrochemical behavior of ISO on the ERGO/GCE was studied and compared with that on bare GCE. The results demonstrated that this ERGO modified GCE showed a high sensitive detection of ISO with good selectivity due to the formation of a stable conjugated $\pi-\pi$ structure between benzene ring of ISO and ERGO. More importantly, the practical application of this novel voltammetric method was demonstrated to determine ISO in tablet, and ISO in plasma after intragastric administration of ISO tablets to SD-rats with satisfied results (Scheme 1). Therefore, the proposed method has a significant attraction both in pharmaceutical control and in pharmacokinetic studies.

\section{Experimental}

\subsection{Reagents and solutions}

Graphite flakes (99.99\%, 325 mesh) was purchased from Alfa Aesar. ISO standards were purchased from Aladdin (Shanghai, China). Xindakang tablets (were $5 \mathrm{mg}$ total flavonoids per tablet) were purchased from local drug store (Sichuan giada pharmaceutical Co., Ltd). Phosphate buffer solution (PBS, purchased from Sinopharm Chemical Reagent Co., Ltd) were prepared by mixing the stock solutions of $0.2 \mathrm{M} \mathrm{NaH}_{2} \mathrm{PO}_{4}$ and $0.2 \mathrm{M} \mathrm{Na}_{2} \mathrm{HPO}_{4}$, and then adjusting the pH with $0.05 \mathrm{M} \mathrm{H}_{3} \mathrm{PO}_{4}$ or $0.05 \mathrm{M} \mathrm{NaOH}$. All reagents were analytical grade and used without further purification. All the solutions were prepared with double-distilled water.

\subsection{Apparatus}

All cyclic voltammetry (CV) and differential pulse voltammetric (DPV) measurements were performed using CHI 630C electrochemical workstation (Shanghai $\mathrm{CH}$ Instruments, China). A conventional three-electrode system was used throughout the experiments, including an ERGO/GCE as the working electrode, a platinum wire as a counter electrode, and an $\mathrm{Ag} / \mathrm{AgCl}$ (saturated) electrode as a reference. Scanning electron microscopy (SEM) was conducted on a Hitachi SU8010 scanning electron microscope for the structural and morphological characterization of the GO (HITACHI, Japan).

\subsection{Preparation of GO}

GO was synthesized from natural graphite powder using a modified Hummers and Offeman's method. ${ }^{25,26}$ In brief, $0.5 \mathrm{~g}$ of graphite flake, $0.5 \mathrm{~g}$ of $\mathrm{NaNO}_{3}$, and $23 \mathrm{~mL}$ of $\mathrm{H}_{2} \mathrm{SO}_{4}$ were stirred together in an ice bath. While maintaining vigorous agitation, an amount of $3 \mathrm{~g}$ of $\mathrm{KMnO}_{4}$ was slowly added, and the rate of addition was controlled carefully to avoid a sudden increase in temperature. The mixture was then maintained at $35{ }^{\circ} \mathrm{C}$ for about $1 \mathrm{~h}$. Deionized water $(40 \mathrm{~mL})$ was gradually added, causing an increase in temperature to $90{ }^{\circ} \mathrm{C}$. Finally, the mixture was further treated with $100 \mathrm{~mL}$ of deionized water and $3 \mathrm{~mL}$ of $30 \% \mathrm{H}_{2} \mathrm{O}_{2}$, turning the color of the solution from dark brown to yellow. GO was then obtained through filtering, water washing, sonicating and drying process.

\subsection{Fabrication of the ERGO/GCE}

The ERGO/GCE was prepared using our previous work. ${ }^{27}$ In brief, GCE (3 mm diameter, CH Instruments, Inc.) was polished successively with 0.3 and $0.05 \mu \mathrm{m} \mathrm{Al}_{2} \mathrm{O}_{3}$ slurry, respectively, and rinsed thoroughly with doubly distilled water between each polishing step. The electrode was then successively sonicated in 1: 1 nitric acid, acetone and doubly distilled water for $2 \mathrm{~min}$, 
respectively. After being cleaned, dried with purified nitrogen stream, $5 \mu \mathrm{L}$ of the GO solution $\left(1 \mathrm{mg} \mathrm{mL}^{-1}\right)$ was drop casted on the surface of well-polished GCE. After being dried, the electrochemically reduced ERGO modified electrode were prepared at reduced potential from 0 to $-1.5 \mathrm{~V}$ for 10 scan cycles by cyclic voltammetry in phosphate buffer (0.1 M pH 4.0).

\subsection{Preparation of standard and sample solution}

The standard solution of $1 \mathrm{mM}$ ISO was prepared by dissolving ISO powder in ethanol, and then it was stored in the dark. Aqueous stock solution of ISO was used for further preparation of final solutions.

Five tablets ( $5 \mathrm{mg}$ per tablet) were finely pulverized, then weighted the average mass and dissolved with $10 \mathrm{~mL}$ ethanol. After sonication and filtration, a suitable aliquot of the clear filtrate was diluted with $\mathrm{pH}$ 4.0 PBS to form appropriated sample solutions.

\subsection{Analytical procedure}

All electrochemical analysis were performed in $10 \mathrm{~mL}$ electrochemical cell containing a certain volume of standard solution or sample solution of ISO, $10 \mathrm{~mL} 0.1 \mathrm{M}$ PBS ( $\mathrm{pH} 4.0$ ) as supporting electrolyte fitted with magnetic stirrer. Then, the CV or DPV curves were recorded. The CV was recorded from 0.2 to $0.5 \mathrm{~V}$ at scan rates from 5 to $500 \mathrm{mV} \mathrm{s}^{-1}$. The DPV was recorded from 0.1 to $0.6 \mathrm{~V}$ with amplitude of $0.05 \mathrm{~V}$, pulse width of $0.05 \mathrm{~s}$, and pulse period of $0.2 \mathrm{~s}$.

\subsection{Application to the pharmacokinetics study in rats}

A pharmacokinetic study was performed in Sprague-Dawley (SD) rats ( $n=6$, weight range $200-250 \mathrm{~g}$ ), which obtained from the Fuzhou Wushi Animal Center. Before experiments, the SD-rats were fasted, and fed with standard laboratory water. The $6 \mathrm{SD}$ rats were set into three groups randomly (2 in each). ISO solution was intragastric administered to each rats at a dose of $50 \mathrm{mg} \mathrm{kg}{ }^{-1}$. The blood samples were collected from rat tail vessels under light post-dosing using microfuge tubes which containing heparin as anti-coagulant before administration and after intragastric administration $(0,0.5 \mathrm{~h}, 1 \mathrm{~h}, 1.5 \mathrm{~h}, 3 \mathrm{~h}, 5 \mathrm{~h}, 6 \mathrm{~h}$, $7 \mathrm{~h}, 8 \mathrm{~h}$, and $24 \mathrm{~h}$ ). Then, the plasma samples were obtained by immediately centrifuging blood samples at $3000 \mathrm{rpm}$ for $10 \mathrm{~min}$ and stored frozen at $-20{ }^{\circ} \mathrm{C}$ until analysis. All plasma samples were analyzed within 1 day. The experimental protocol was approved by the Institutional Animal Ethics Committee (IAEC) for the use of experimental animals and all animal studies were carried out according to the Guide for Care and Use of Laboratory Animals.

\section{Results and discussion}

\subsection{Choice of the materials}

To study the clinical pharmacokinetic of ISO by electrochemical method, it is necessary to select a highly sensitive, rapid and stable detection method. For this purpose, various materials have been investigated to develop the electrochemical sensor interface, such as electrochemically reduced graphene oxide (ERGO), CdS QDs quantum dots (QDs), $\mathrm{TiO}_{2}$ nanomaterial, $\mathrm{MoS}_{2}$ nanosheets and $\mathrm{Au}$ nanoclusters (Fig. S1†). The results indicated that the ERGO modified GCE exhibited the most stable and sensitive signal. We rationalize this result as the good biocompatibility, electrical conductivity, stability, and large specific surface area of the ERGO. Besides, the $\pi-\pi$ effect between ERGO and ISO could facilitate the enrichment of the

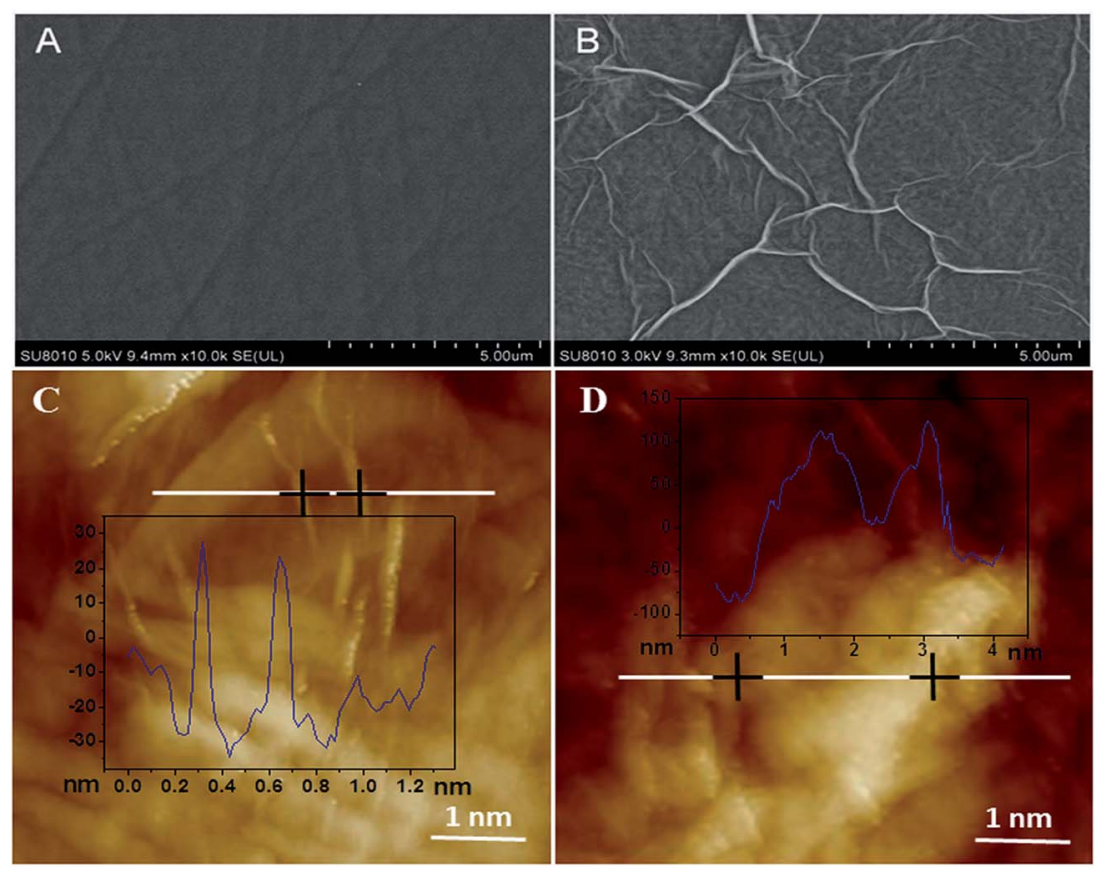

Fig. 1 SEM images of bare GCE (A) and ERGO modified GCE (B); AFM images of ERGO modified GCE (C) and after enrichment of ISO (D). 
ISO on the electrode surface, which greatly improved the sensitivity of the sensor. Therefore, in this experiment, ERGO/ GCE was chosen to study the pharmacokinetics of ISO.

\subsection{Characterization of the modified electrode}

SEM was used to investigate the surface morphologies of bare GCE and ERGO/GCE. As can be seen from Fig. 1A, the bare GCE surface was rather smooth and flat, and the shape of graphene on the ERGO/GCE surface was planar sheet-like, indicating that the ERGO was successfully casted on the bare GCE surface (Fig. 1B). On the other hand, the AFM images showed that after the ISO enriched on the surface of the ERGO/GCE, the thickness of the modified electrode was significantly increased (Fig. 1C and D).

\subsection{Electrochemical behavior of ISO on the ERGO/GCE}

The electrochemical response of ISO at the bare GCE and the ERGO/GCE were examined by CVs. As shown in Fig. 2A, no redox peaks were observed on both the bare GCE (curve a) and ERGO/GCE (curve c) in blank buffer solution. When ISO was added into the blank solution, a tiny pair of redox peaks appeared $\left(E_{\mathrm{pa}}=0.399 \mathrm{~V}, E_{\mathrm{pc}}=0.359 \mathrm{~V}\right.$ ) at the bare GCE (curve b). As expected, for the ERGO/GCE (curve d, $E_{\mathrm{pa}}=0.372 \mathrm{~V}, E_{\mathrm{pc}}=$
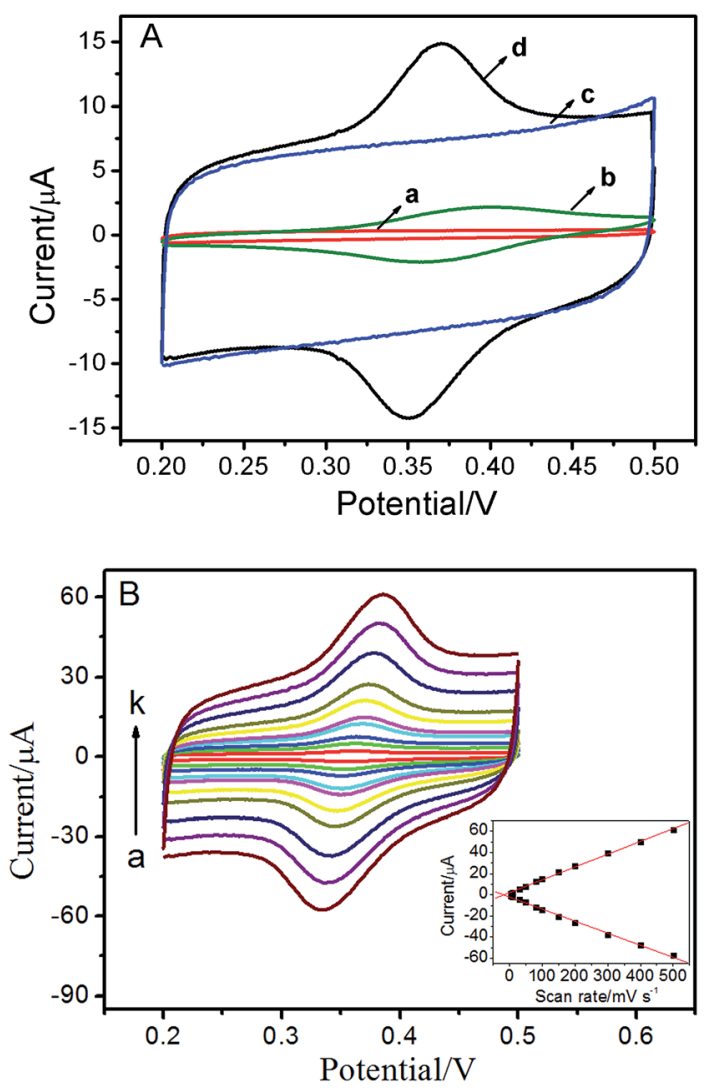

Fig. 2 (A) CVs on bare GCE (a, b) and ERGO/GCE (c, d) in the absence $(a, c)$ and in the presence $(b, d)$ of $5 \times 10^{-6} \mathrm{MISO}$ in pH 4.0 PBS. (B) CVs of ISO on ERGO modified electrode at different scan rate of 5, 10, 30, $50,80,100,150,200,300,400$ and $500 \mathrm{mV} \mathrm{s}^{-1}$. Inset: plot of peak currents vs. scan rates.
$0.349 \mathrm{~V})$, the currents of the redox peaks $\left(I_{\mathrm{pa}, \mathrm{ERGO} / \mathrm{GCE}}=7.16 \mu \mathrm{A}\right.$, $\left.I_{\mathrm{pc}, \mathrm{ERGO} / \mathrm{GCE}}=7.01 \mu \mathrm{A}\right)$ increased remarkably compared with bare GCE $\left(I_{\mathrm{pa}, \mathrm{GCE}}=1.71 \mu \mathrm{A}, I_{\mathrm{pc}, \mathrm{GCE}}=1.58 \mu \mathrm{A}\right)$, which could be attributed to the high specific surface area and good conductivity of ERGO. The scan rate is probably the most important experimental parameter for evaluating the effects due to adsorbed reactant and/or due to reactant reaching the electrode via diffusion. With the increase of the scan rates, the redox peak currents of ISO increase simultaneously, accompanied with an enlargement of the peak separation (Fig. 2B). Moreover, both the anodic and cathodic peak currents increase linearly with the scan rates between 5 and $500 \mathrm{mV} \mathrm{s}^{-1}$ (inset of Fig. 2B). These results suggested that the electron-transfer was relatively fast and the reaction of this reversible couple is a surface adsorption-controlled process of the ISO, which is in agreement with the previous report. ${ }^{15}$

\subsection{Optimization of experimental conditions}

The effect of $\mathrm{pH}$ values on the redox reaction of ISO on the ERGO/GCE was also investigated in the $\mathrm{pH}$ range from 3.0 to 9.0. In Fig. 3A, the oxidation peak current of ISO increased with increasing $\mathrm{pH}$ value until it reaches 4.0, and then decreases when the $\mathrm{pH}$ increases further, which is in agreement with previous reports. ${ }^{15,28}$ Considering the sensitivity for determining ISO, pH 4.0 is chosen for the subsequent analytical experiments. What is more, with $\mathrm{pH}$ value of the solution increasing,
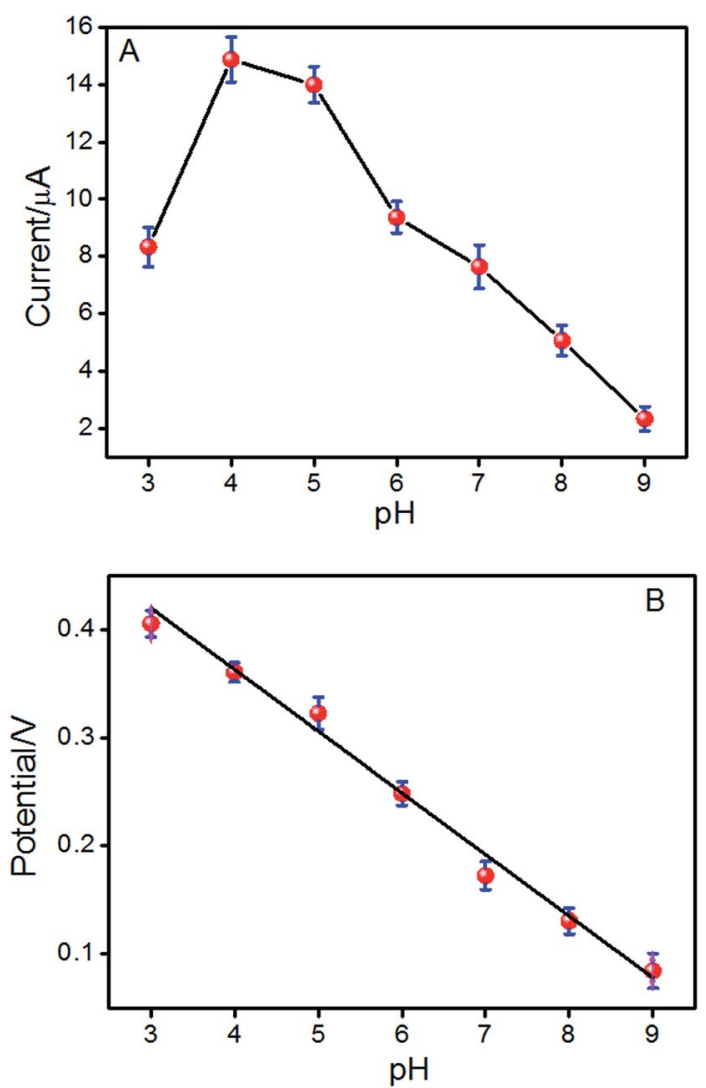

Fig. 3 The effect of $\mathrm{pH}$ on the peak current $(A)$ and peak potential $\left(E_{\mathrm{pa}}\right)$ (B) of the reversible couple for ISO in phosphate buffer solutions. 

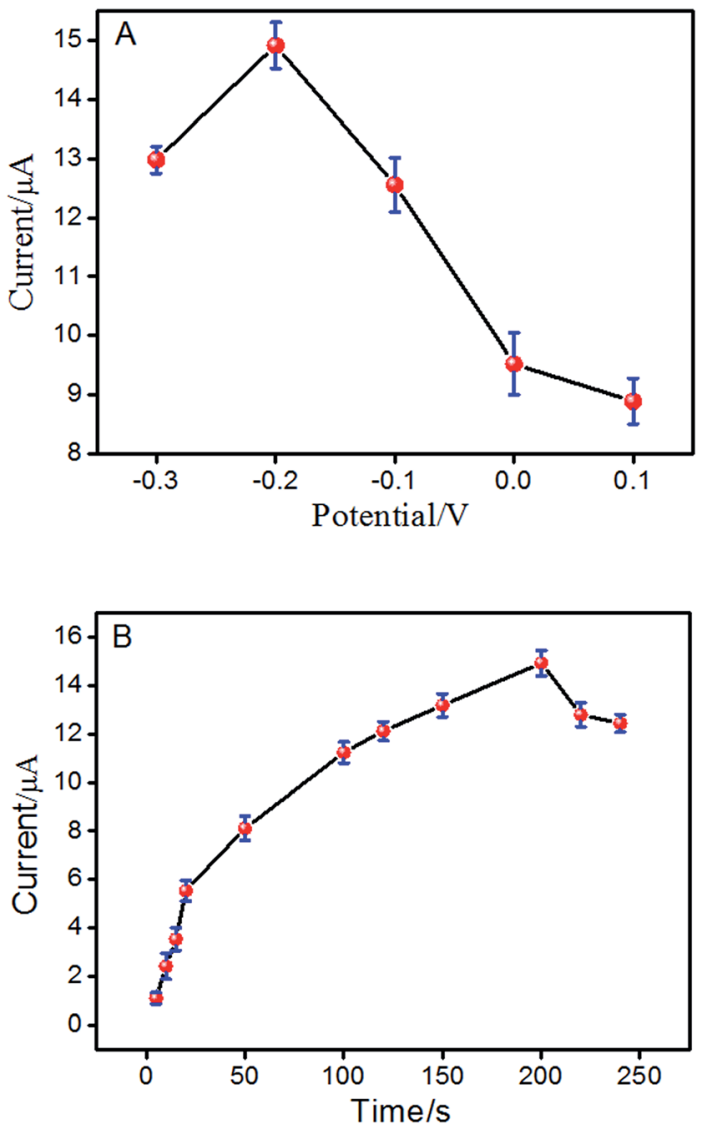

Fig. 4 Effect of the preconcentration potential (A) and preconcentration time (B) on peak current of ISO by DPV in pH 4.0 PBS.

the oxidation peak potential $\left(E_{\mathrm{pa}}\right)$ shifts negatively, indicating that protons have taken part in the electrode reaction process of ISO. ${ }^{29}$ It is found that the value of the oxidation peak potential changes linearly with $\mathrm{pH}$ values in the range of 3.0-9.0 with a slop of $-56.2 \mathrm{mV} \mathrm{pH}^{-1}$ (Fig. 3B), which is approximately close

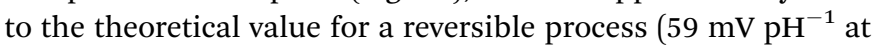
$298 \mathrm{~K}$ ), suggesting that the number of protons and electrons involved in the electrochemical redox process of ISO is equal. ${ }^{\mathbf{1 4}}$

Hence, the effect of different accumulation conditions on the electrochemical response of ISO at ERGO/GCE was also studied. Fig. $4 \mathrm{~A}$ reveals that at the accumulation potential of $-0.2 \mathrm{~V}$, the maximum peak current was reached. Thus, the optimal adsorption potential was performed at $-0.2 \mathrm{~V}$. In addition, the effect of accumulation time on the oxidation peak current of ISO was also investigated with varying the accumulation time with a $-0.2 \mathrm{~V}$ accumulation potential. As shown in Fig. $4 \mathrm{~B}$, the peak current increased rapidly with the accumulation time and reached a maximum at $200 \mathrm{~s}$. Then, it decreased rapidly with increased accumulation time. Thus, the preconcentration time of $200 \mathrm{~s}$ has been used in the following measurements.

\subsection{Linearity, detection limit and reproducibility}

Under the optimum conditions mentioned above, the determination of ISO using the ERGO/GCE could be achieved. As shown in the Fig. 5, the peak currents were linearly proportional to ISO concentrations in the ranges of $1.0 \times 10^{-8}$ to $1.2 \times$ $10^{-5} \mathrm{M}, I=5.198 \mathrm{C}+2.669(r=0.9902)$. The detection limits for ISO was $4.3 \times 10^{-9} \mathrm{M}$. The linear range and detection limit of this method was compared with other methods reported
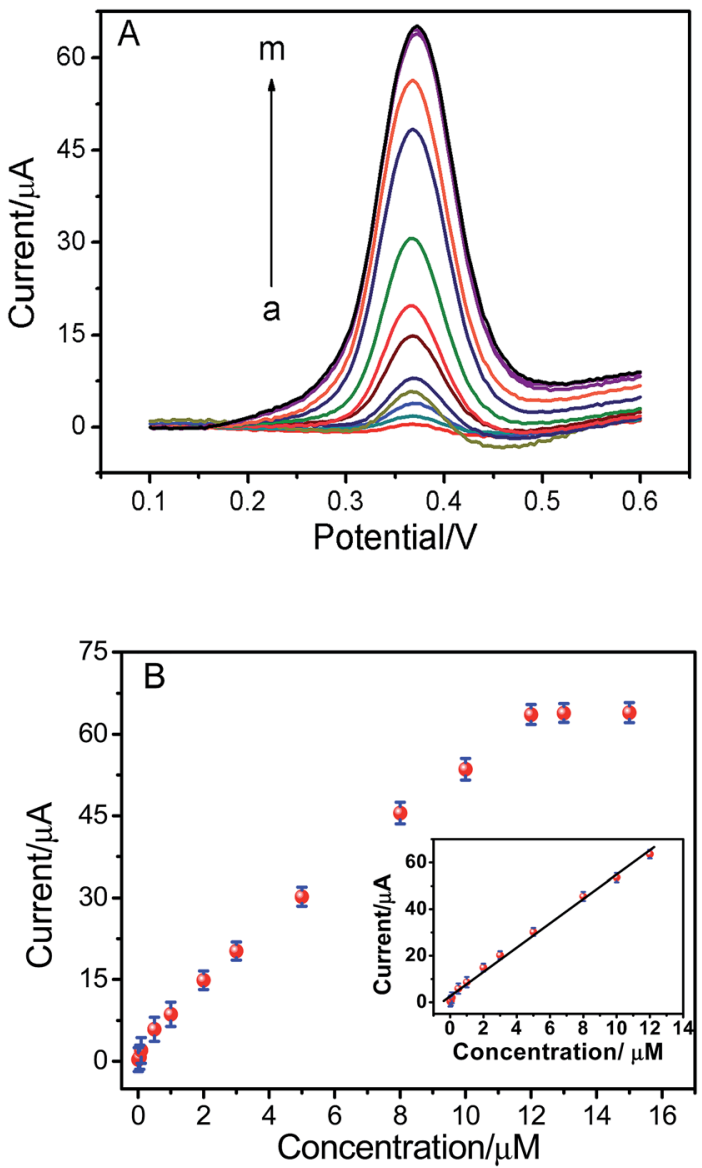

Fig. 5 (A) DPVs of different ISO concentrations at ERGO/GCE. ISO concentrations (from a to $\mathrm{m}$ : 0.01, 0.05, 0.1, 0.5, 1.0, 2.0, 3.0, 5.0, 8.0, 10.0, 12.0, 13.0 and $15.0 \mu \mathrm{M}$ ). (B) The relationship between ISO concentrations and peak current.

Table 1 Comparison of different methods for the detection of ISO

\begin{tabular}{llll}
\hline Methods & Linear range & Detection limits & Ref. \\
\hline Electrochemistry & $\begin{array}{l}1 \times 10^{-8} \text { to } \\
1 \times 10^{-7} \mathrm{M},\end{array}$ & $5.0 \times 10^{-9} \mathrm{M}$ & 15 \\
& $1 \times 10^{-6}$ to & & \\
& $1.0 \times 10^{-5} \mathrm{M}$ & & \\
UPLC-MS $^{a}$ & $0.01-1.0 \mu \mathrm{g} \mathrm{mL}$ & $3.48 \mathrm{ng} \mathrm{mL}^{-1}$ & 30 \\
GC-MS $^{b}$ & - & $2.5 \mu \mathrm{mL}^{-1}$ & 4 \\
RP-HPLC $^{c}$ & $0.008-0.400 \mathrm{mg} \mathrm{mL}^{-1}$ & $2.33 \mu \mathrm{gL} \mathrm{m}^{-1}$ & 5 \\
RP-HPLC $_{\text {Electrochemistry }}$ & $0.425-17.008 \mu \mathrm{mL}^{-1}$ & $0.061 \mu \mathrm{gL}^{-1}$ & 31 \\
& $1.0 \times 10^{-8} \mathrm{to}$ & $4.3 \times 10^{-9} \mathrm{M}$ & This work \\
& $1.2 \times 10^{-5} \mathrm{M}$ & &
\end{tabular}

${ }^{a}$ Ultra-performance liquid chromatography-mass spectrometry. ${ }^{b}$ Gas chromatography-mass spectrometry. ${ }^{c}$ Reversed phase high performance liquid chromatography. 


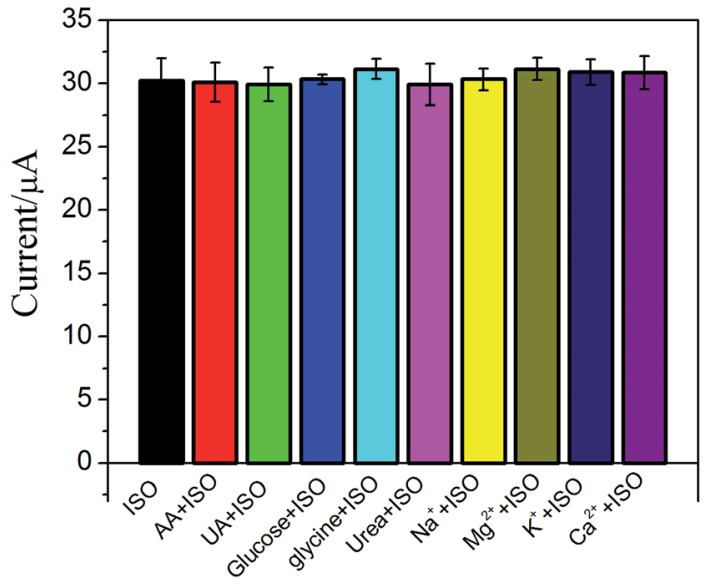

Fig. 6 The effect of different interfering substances on electrical signals.

previously (Table 1), the results indicated that the analytical performance of this sensor was better than that of the other existing sensors for detection of ISO. Thus, this method not only expanded the application of ERGO, but also opened new doors toward the rapid detection of ISO. The relative standard deviation of six repetitive measurements of standard solution containing $1 \times 10^{-6} \mathrm{M}$ ISO is $2.53 \%$, showing good reproducibility.

\subsection{Interference study}

The effect of possible interfering species such as uric acid (UA), ascorbic acid (AA), glycine and some ions on ISO detection has been examined (Fig. 6). No significant interfering was observed upon addition of $0.1 \mathrm{mM} \mathrm{UA}, \mathrm{AA}$, glucose, glycine, $\mathrm{K}^{+}, \mathrm{Mg}^{2+}$, $\mathrm{Ca}^{2+}$ and $\mathrm{Na}^{+}$in $5 \mu \mathrm{M}$ ISO. These results suggested that the proposed ERGO/GCE based sensor has good selectivity.

\subsection{The recovery of the method}

The method presented above was applied to the determination of ISO in tablets by DPV method described here. The results were listed in the Table 2. It shows that the proposed method has an acceptable recovery and could be used to determine ISO in tablets.

\subsection{Study of pharmacokinetics}

To demonstrate the utility of the present method in vivo conditions, the pharmacokinetics of ISO in the plasma of SD

Table 2 Determination of ISO in tablet ( $n=6$, repetitive measurement number)

\begin{tabular}{llllll}
\hline Analyst & $\begin{array}{l}\text { Labeled } \\
(\mathrm{mg})\end{array}$ & $\begin{array}{l}\text { Added } \\
(\mathrm{mg})\end{array}$ & Found $(\mathrm{mg})$ & $\begin{array}{l}\text { R.S.D. } \\
(\%)\end{array}$ & $\begin{array}{l}\text { Recovery } \\
(\%)\end{array}$ \\
\hline \multirow{2}{*}{ ISO } & 3.74 & 0 & $3.74 \pm 0.07$ & 1.2 & - \\
& & 1 & $4.63 \pm 0.09$ & $3.3 \%$ & $89 \%$ \\
& & 1.5 & $5.04 \pm 0.11$ & $2.5 \%$ & $104 \%$ \\
& & 2 & $6.00 \pm 0.17$ & $2.9 \%$ & $113 \%$
\end{tabular}
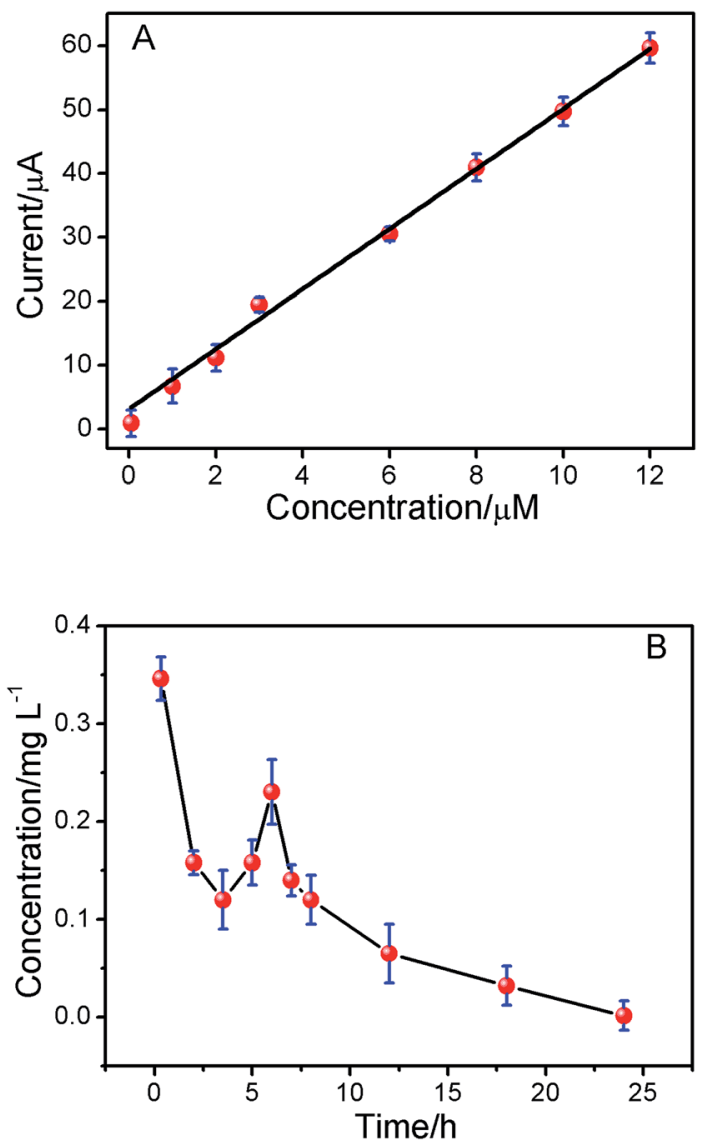

Fig. 7 (A) The relationship between ISO concentration $(0.05,1.0,2.0$, 3.0, 6.0, 8.0, 10.0 and $12.0 \mu \mathrm{M}$ ) in plasma and peak current. (B) The concentration-time curve of ISO after intragastric administration in rat.

rats was studied. The oxidation peak currents of ISO spiked in blank plasma as measured by DPV increased linearly with the concentration of ISO in the range of $5.0 \times 10^{-8}$ to $1.2 \times 10^{-5} \mathrm{M}$ (Fig. 6A, $r=0.9956)$. The present method was applied to the pharmacokinetic investigations of ISO in SD-rats plasma after intragastric administration. The mean plasma concentrationtime curve profile was illustrated in Fig. 7B. Pharmacokinetic

Table 3 Pharmacokinetic parameters of ISO in SD rats' plasma after administering ISO solutions (50 $\left.\mathrm{mg} \mathrm{kg}^{-1}, n=6\right)^{a}$

SD rat estimate (mean \pm S.D.)

Parameters

$1.684 \pm 0.23$

$T_{1 / 2 \alpha}(\mathrm{h})$

$6.728 \pm 0.37$

$T_{1 / 2 \beta}(\mathrm{h})$

$C_{\max }\left(\mathrm{mg} \mathrm{L}^{-1}\right)$

$0.346 \pm 0.29$

$\mathrm{AUC}_{0-\infty}\left(\mathrm{mg} \mathrm{L}^{-1} \mathrm{~h}^{-1}\right)$

$4.119 \pm 0.33$

$\mathrm{AUMC}_{0-t}$

$\mathrm{MRT}_{0-t}(\mathrm{~h})$

$5.121 \pm 0.039$

$3.633 \pm 0.28$

${ }^{a} T_{1 / 2 \alpha}$, distribution half-life; $T_{1 / 2 \beta}$, elimination half-life; $C_{\max }$, maximum plasma concentration; $\mathrm{AUC}_{0-\infty}$, areas under the curve; $\mathrm{AUMC}_{0-t}$, the area under the first moment of the plasma concentration-time curve; $\mathrm{MRT}_{0-t}$, mean residence time. 
parameters were estimated by Kinetica version 4.4.1 software (Thermo Electron Corporation, USA) and listed in Table 3. As shown in Fig. 7B, there was a secondary absorption peak appeared on 7 hours due to the enterohepatic circulation, which was in accordance with the previous reports by LC-MS/MS. ${ }^{32}$ The results indicated that the method is applicable to pharmacokinetic studies of ISO.

\section{Conclusions}

A sensitive and rapid electrochemical assay method to study the pharmacokinetics of ISO based on ERGO/GCE was successfully established. The present electrochemical assay approach improved the detection limit and provided a wide range of concentrations for determination of ISO. Furthermore, the validated methods were successfully applied to study the pharmacokinetic of ISO in SD rat after intragastric administration. The present method is rapid, easy, low cost, and showed satisfactory reproducibility, good stability, excellent sensitivity, and acceptable recovery. Thus, this method could be a promising approach to the clinical pharmacokinetics research as well as for intensive medicines monitoring.

\section{Acknowledgements}

We gratefully acknowledge the financial support of the National Natural Science Foundation of China (21405015), Joint Funds for the Innovation of Science and Technology, Fujian Province (2016Y9055, 2016Y9054), the Natural Science Foundation of Fujian Province of China (2014J07009), and the Medical Elite Cultivation Program of Fujian (2013-ZQN-JC-25).

\section{References}

1 K. A. Manu, M. K. Shanmugam, L. Ramachandran, F. Li, K. S. Siveen, A. Chinnathambi, M. E. Zayed, S. A. Alharbi, F. Arfuso, A. P. Kumar, K. S. Ahn and G. Sethi, Cancer Lett., 2015, 363, 28-36.

2 A. Carotenuto, E. Fattorusso, V. Lanzotti, S. Magno, V. De Feo and C. Cicala, Phytochemistry, 1997, 44, 949-957.

3 F. Pellati, F. P. Precipe, D. Bertelli and S. Benvenuti, J. Pharm. Biomed. Anal., 2013, 81-82, 126-132.

4 F. X. Deng and S. W. Zito, J. Chromatogr. A, 2003, 986, 121127.

5 Y. G. Zu, C. Y. Li, Y. J. Fu and C. J. Zhao, J. Pharm. Biomed. Anal., 2006, 41, 714-719.

6 P. Gebauer, D. Malá and P. Boček, Electrophoresis, 2009, 30, 29-35.

7 B. Ma, X. M. Zhou, G. Wang, H. Q. Huang, Z. P. Dai, J. H. Qin and B. C. Lin, Electrophoresis, 2006, 27, 904-909.
8 S. A. Lim and M. U. Ahmed, RSC Adv., 2016, 6, 24995-25014. 9 S. T. Sultana, J. T. Babauta and H. Beyenal, Biofouling, 2015, 31, 745-758.

10 H. Wang and Z. J. Ren, Biotechnol. Adv., 2013, 31, 1796-1807.

11 P. Xiao, Q. Zhou, F. Xiao, F. Q. Zhao and B. Z. Zeng, Int. J. Electrochem. Sci., 2006, 1, 228-237.

12 A. L. Liu, S. B. Zhang, L. Y. Huang, Y. Y. Cao, H. Yao, W. Chen and X. H. Lin, Chem. Pharm. Bull., 2008, 56, 745-748.

13 S. Sun, M. Q. Zhang, Y. J. Li and X. W. He, Sensors, 2013, 13, 5493-5506.

14 D. B. Lu, S. X. Lin, L. T. Wang, T. Li, C. M. Wang and Y. Zhang, J. Solid State Electrochem., 2014, 18, 269-278.

15 A. L. Liu, S. B. Zhang, W. Chen, L. Y. Huang, X. H. Lin and X. H. Xia, Talanta, 2008, 77, 314-318.

16 A. T. Lawal, Talanta, 2015, 131, 424-443.

17 F. H. Cincotto, D. L. C. Golinelli, S. A. S. Machado and F. C. Moraes, Sens. Actuators, B, 2017, 239, 488-493.

18 J. H. Xu, Y. Z. Wang and S. S. Hu, Microchim. Acta, 2017, 184, 1-44.

19 D. Chen, H. B. Feng and J. H. Li, Chem. Rev., 2012, 112, 60276053.

20 M. Thakur, M. K. Kumawat and R. Srivastava, RSC Adv., 2017, 7, 5251-5261.

21 Y. H. Zheng, L. Huang, Z. Y. Zhang, J. Z. Jiang, K. Y. Wang, L. M. Peng and G. Yu, RSC Adv., 2017, 7, 1776-1781.

22 Y. Song, Y. Luo, C. Z. Zhu, H. Li, D. Du and Y. H. Lin, Biosens. Bioelectron., 2016, 76, 195-212.

23 W. Zhou, K. Y. Tam, M. X. Meng, J. J. Shan, S. C. Wang, W. Z. Ju, B. C. Cai and L. Q. Di, J. Chromatogr. A, 2015, 1376, 84-97.

24 G. W. Li, X. L. Zeng, Y. Xie, Z. Z. Cai, J. C. Moore, X. R. Yuan, Z. H. Cheng and G. Ji, Fitoterapia, 2012, 83, 182-191.

25 W. S. Hummers and R. E. Offeman, J. Am. Chem. Soc., 1958, 80, 1339.

26 L. J. Cote, F. Kim, J. X. Huang and L. Blodgett, J. Am. Chem. Soc., 2009, 131, 1043-1049.

27 H. L. Guo, X. F. Wang, Q. Y. Qian, F. B. Wang and X. H. Xia, ACS Nano, 2009, 3, 2653-2659.

28 S. V. Jovanovic, S. Steenken, M. Tosic, B. Marjanovic and M. G. Simic, J. Am. Chem. Soc., 1994, 116, 4846-4851.

29 H. R. Zare, Z. Sobhani and M. Mazloum-Ardakani, Sens. Actuators, B, 2007, 126, 641-647.

30 G. W. Li, X. L. Zeng, Y. Xie, Z. Z. Cai, J. C. Moore, X. R. Yuan, Z. H. Cheng and G. Ji, Fitoterapia, 2012, 83, 182-191.

31 M. Olszewska, J. Pharm. Biomed. Anal., 2008, 48, 629-635.

32 J. He, Y. Feng, H. Z. Ouyang, B. Yu, Y. X. Chang, G. X. Pan, G. Y. Dong, T. Wang and X. M. Gao, J. Pharm. Biomed. Anal., 2013, 84, 189-195. 